\title{
Acceso radial durante la angioplastía primaria en el infarto agudo al miocardio
}

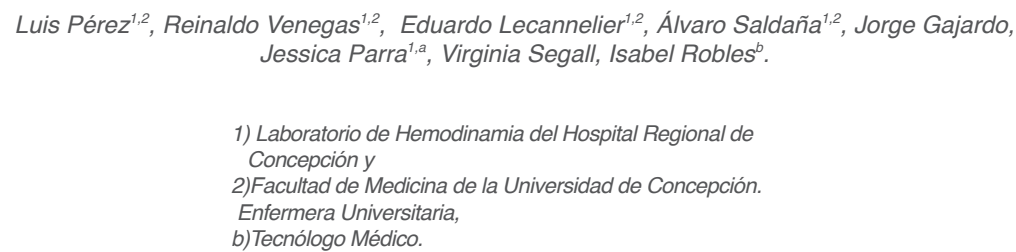

\section{Resumen:}

Introdución: El acceso radial (AR) ha demostrado reducir las complicaciones vasculares asociadas a la coronariografía y angioplastía coronaria; sin embargo, su rol en la angioplastía primaria (AP) durante el infarto agudo al miocardio con supradesnivel del segmento ST (IAM c/SDST) es muy escasa. En diciembre de 2007, nuestro equipo adoptó esta técnica como primera opción durate la AP.

Objetivo. Estudiar si el AR durante la AP es tan rápido y seguro como el acceso femoral (AF).

Métodos: Utilizando el registro de nuestro laboratorio, analizamos todos los pacientes con IAM c/SDST que fueron tratados con AP en nuestro hospital en el período Septiembre 2005 - Agosto 2009, y luego de identificar los pacientes de acuerdo al AF y AR, comparamos el tiempo "puerta-balón", los resultados angiográficos, el éxito clínico y las complicaciones rela- cionadas al acceso. El seguimiento se efectuó a través de las visitas médicas y vía telefónica. Los valores se muestran como promedio \pm DS.

Resultados: En el periodo estudiado, en 354 (75\%) pacientes se utilizó AF y en 118 (25\%) el AR. La edad promedio de ambos grupos fue similar, $61 \pm 12$ años en el grupo con $\mathrm{AF}$ vs $62 \pm 12$ años en $\mathrm{AR}(\mathrm{p}=\mathrm{ns})$ y no hubo diferencias en las características clínicas de los grupos. El tiempo "puerta-balón" fue $62 \pm 37$ minutos vs $61 \pm 29$ minutos en AF y AR respectivamente ( $p=n s$ ) con una tasa de éxito superior al 94\% en ambo grupos. En el grupo de AF hubo 3\% de complicaciones vasculares vs $0 \%$ en AR. La mortalidad global a 30 días fue $5,1 \%$ en el grupo de AF y de 4,0\% en AR (p=ns).

Conclusión: El acceso radial para la AP permite abrir la arteria culpable en tiempos similares al AF y con una menor incidencia de complicaciones vasculares.

\footnotetext{
Correspondencia:

Dr. Luis A. Pérez Pino

San Martín 1436, Laboratorio de Hemodinamia,

Hospital Gmo. Grant Benavente, Concepción

e-mail: I.perezp@yahoo.es

Fono: $56-41-2722640$
} 


\section{Radial artery access to perform primary angyoplasty in acute myocardial infarction}

Background: Radial access (RA) has been shown to help reduce vascular complications of coronary arteriography and angioplasty (PTCA). However, little experience has been reported with RA to perform primary PTCA in ST elevation acute myocardial infarction (STEMI). Since December 2007, we selected RA as a first option for primary PTCA in STEMI.

Aim: to determine whether RA is as effective, safe and expeditious as femoral access (FA) in primary PTCA for STEMI

Method: we performed a retrospective review of our database of all patients undergoing primary PTCA in our laboratory from Sept 2005 through August 2009. We compared all patients who had a PTCA using a FA to those in whom the RA was used. The door to balloon time, angiographic results, clinical success rate and complications related to the vascular access were compared between groups. Follow-up

\section{Introducción}

La angioplastía primaria (AP) es el método de elección en el tratamiento del infarto agudo del miocardio con supradesnivel del segmento ST (IAM c/SDST), reduce de manera significativa la mortalidad y se asocia a una menor incidencia de reinfarto y de complicaciones hemorrágicas ${ }^{1,2}$. En los últimos años se ha buscado la forma de mejorar los resultados de la angioplastía y parte de ello ha sido el desarrollo de nuevos antitrombóticos y antiplaquetarios ${ }^{3}$, los que han demostrado reducir los eventos isquémicos recurrentes y las complicaciones hemorrágicas, pero asociados a un significativo aumento en los costos de los procedimientos. Por otro lado hay estudios que demuestran que la utili- was based con clinical visits and phone calls. Values are expressed as mean and SD.

Results: 375 patients had an FA (75\%) and 118 an RA (25\%). Mean age was similar in both groups ( $61 \pm 12$ vs $62 \pm 12$, respectively, NS). Selected clinical characteristics did no differ between groups. Door to balloon time was $62 \pm 37 \mathrm{~min}$ for FA and $61 \pm 29 \mathrm{~min}$ for RA (NS). Clinical success rate exceeded $94 \%$ in both groups. Vascular complications occurred in $3 \%$ in the AF group. No vascular complications were observed in the RA group. Thirty day overall mortality rates were $5.2 \%$ in FA and $4.0 \%$ in RA (NS).

Conclusion: Radial access can be used for primary PTCA in STEMI with similar success rates and lower incidence of vascular complications, when compared to the traditional femoral access.

Key words: radial access, PTCA, STEMI

zación del acceso radial (AR) para la angioplastía coronaria posee una menor incidencia de complicaciones hemorrágicas ${ }^{4-6}$, lo que derivaría en una menor mortalidad en comparación con el acceso femoral (AF). Sin embargo, las experiencias en AP son limitadas y se cuestiona que la vía radial pudiese retardar el tiempo "puerta-balón".

Nuestro objetivo es demostrar que el AR durante el IAM es tan efectivo y seguro como la vía femoral para obtener una rápida reperfusión coronaria.

\section{Método}

Realizamos un análisis retrospectivo de la base de datos que incluyó a todos los pacientes con IAM c/SDST 
que ingresaron al Servicio de Urgencia de nuestro Hospital entre Septiembre 2005 y Agosto de 2009 y que fueron tratados con angioplastía primaria.

Fueron sometidos a estudio angiográfico de urgencia los pacientes que presentaban dolor toráxico o un equivalente anginoso asociado a elevación del segmento ST en dos derivaciones contiguas o un nuevo bloqueo completo de la rama izquierda e idealmente llevar menos de 12 horas de evolución. En la mayoría de los pacientes el acceso fue la arteria femoral, sin embargo, en Diciembre de 2007 instauramos el acceso radial.

Se definen, de manera no aleatorizada, los grupos de acuerdo al acceso utilizado, AR y AF en los que se comparó la edad, sexo, los factores de riesgo cardiovascular tales como tabaquismo, hipertensión arterial, diabetes mellitus y dislipidemia, el tiempo "puerta-balón”, los resultados angiográficos y clínicos. Además se comparó la incidencia de complicaciones vasculares y la dosis de radiación.

El acceso arterial fue elegido a gusto del operador y de acuerdo a las características anatómicas de los pacientes, tales como pulsos periféricos, test de Allen y/o características del pulso radial. Los pacientes con AR recibieron verapamilo $5 \mathrm{mg}$ intraradial directo.

El tiempo puerta-balón fue definido como el tiempo comprendido entre que el paciente es evaluado por el médico del servicio de urgencias con un ECG compatible y el primer inflado del balón de angioplastía.

Se consideró éxito angiográfico cuando hubo estenosis residual $<20 \%$ y flujo TIMI 3. Se definió mortalidad precoz como aquella sucedida antes de los 30 días. Reinfarto fue definido por la presencia de un nuevo episodio de dolor o su equivalente anginoso, asociado a elevación enzimático, con o sin cambios electrocardiográficos.

Todos los pacientes recibieron 300 o $500 \mathrm{mg}$ de aspirina por vía oral en la sala de urgencia y durante la angioplastía coronaria a todos se les administró una dosis de heparina intravenosa (100 U por kilo de peso). Finalizada la angioplastía todos recibieron una carga oral de 300 mg Plavix ${ }^{\circledR}$ y luego se les indicó 75 mg/ día por un mes. En esta serie, tres pacientes recibieron algún bloqueador de GP IIb/IIIa.

El seguimiento fue realizado a través de los controles médicos con ficha clínica y mediante el contacto telefónico.

En el análisis estadístico los resultados se presentan como promedio y desviación estándar y para las comparaciones se realiza un test exacto de Fisher.

\section{Resultados}

En el período analizado, 472 pacientes con IAM c/ SDST fueron tratados con AP. En 354 (75\%) se utilizó la vía femoral y en 118 (25\%) la arteria radial (derecha o izquierda). Desde el inicio de la "era radial" en diciembre de 2007, 108 pacientes con IAM fueron tratados usando la vía lo que correspondió al 47\%, en los otros 118 se usó el acceso radial.

El 76\% de los pacientes ingresó con infarto de menos de 6 horas de evolución desde el inicio de los síntomas, $16 \%$ entre 6 y 12 horas y el $8 \%$ restante lo hizo con un infarto de más de 12 horas de evolución.

En el $46 \%$ de los casos el infarto fue de pared anterior, el $45 \%$ de pared inferior y el $9 \%$ restante fue de pared lateral. La principal arteria tratada fue la descendente anterior (45\%), seguida por la arteria coronaria derecha y la fracción de eyección promedio fue $59 \pm 12 \%$. El rango de edad del grupo total fue 23-89 años y la edad promedio de ambos grupos fue similar, $61 \pm 12$ años en el grupo con AF vs $62 \pm 12$ años en AR ( $\mathrm{p}=\mathrm{ns})$. No hubo diferencias en los factores de riesgo (Tabla 1).

\begin{tabular}{|l|l|l|l|}
\hline \multicolumn{4}{|l}{ Tabla 1. Características Clínicas de los pacientes $(\mathrm{n}=472)$} \\
\hline Variable & $\begin{array}{c}\text { Femoral } \\
\mathrm{n}=354\end{array}$ & $\begin{array}{c}\text { Radial } \\
\mathrm{n}=118\end{array}$ & $\mathrm{n}$ \\
\hline Edad, años (23-92) & $61 \pm 12$ & $62 \pm 12$ & $\mathrm{~ns}$ \\
\hline Hombres & $260(73)$ & $91(77)$ & $\mathrm{ns}$ \\
\hline Hipertensión & $185(52)$ & $70(59)$ & $\mathrm{ns}$ \\
\hline Diabetes mellitus & $64(18)$ & $26(22)$ & $\mathrm{ns}$ \\
\hline Dislipidemia & $38(11)$ & $9(8)$ & $\mathrm{ns}$ \\
\hline Hábito tabáquico & $97(27)$ & $25(21)$ & $\mathrm{ns}$ \\
\hline
\end{tabular}


Hubo éxito en el acceso en el $100 \%$ del grupo que utilizó la vía femoral, en cambio, en el 3\% del grupo con acceso radial fue necesario la conversión a vía femoral, principalmente debido a la presencia de loop en la arteria.

El tiempo entre la punción arterial y el inflado del balón fue 7,2 minutos en AF vs 6,2 minutos en $\mathrm{AR}$ (p=ns) y los tiempos puerta-balón fueron $62 \pm 37$ minutos vs $61 \pm 29$ minutos en AF y AR respectivamente $(\mathrm{p}=\mathrm{ns})$ (Figura 1).

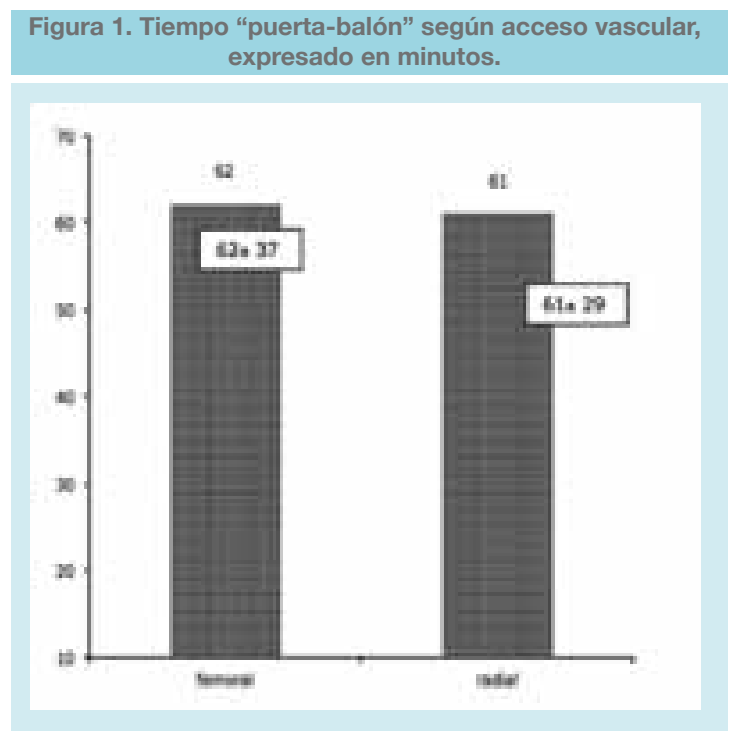

Hubo éxito angiográfico en $94 \%$ del AF y $95 \%$ en AR. En ambos casos la principal causa de fracaso fue la persistencia de trombos intracoronarios.

La radiación durante el procedimiento fue discretamente superior con al AR $(1405 \pm 926$ mGy para en $\mathrm{AR}$ versus $1219 \pm 953 \mathrm{mGy}$ para el $\mathrm{AF}, \mathrm{p}=0,5)$. De igual manera en el grupo de pacientes con AR hubo un discreto pero significativo mayor volumen de medio de contraste utilizado $(168 \pm 77$ vs $132 \pm 48 \mathrm{ml}, \mathrm{p}<0,005)$.
No se observaron complicaciones vasculares en los pacientes con AR en cambio 3\% de los pacientes con AF presentaron una complicación, la mayoría de los cuales fueron hematomas leves a moderados, sin embargo esta diferencia no es estadísticamente significativa. En un caso hubo necesidad de reparación vascular quirúrgica. (Figura 2)

La mortalidad global a 30 días fue $5,1 \%$ en el grupo de AF y de 4,0\% en AR ( $\mathrm{p}=\mathrm{ns})$.

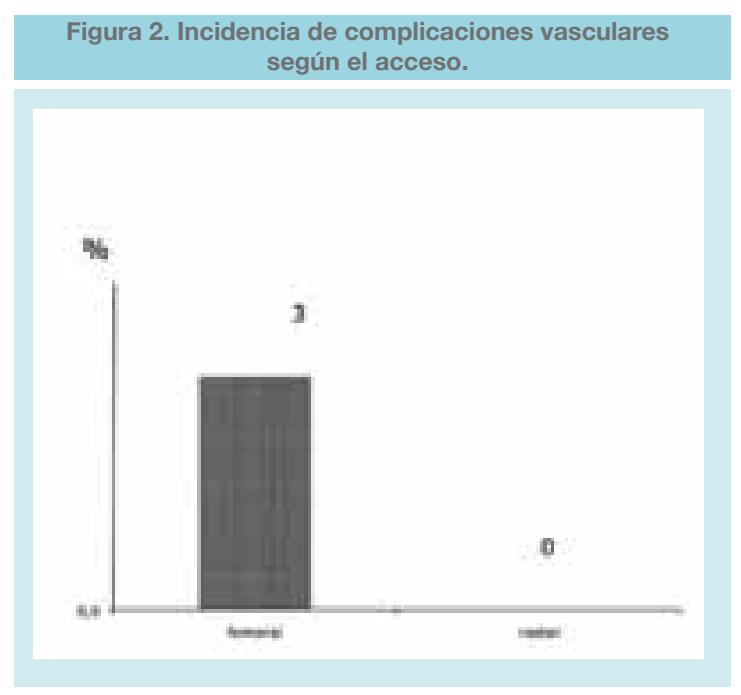

\section{Discusión}

El principal hallazgo de nuestro estudio, es que la utilización del acceso radial durante la AP en el IAM c/ SDST es un método igual de efectivo que la vía femoral y que conllevaría una menor incidencia de complicaciones vasculares sin retrasar el tiempo en lograr la apertura de la arteria ocluida.

Numerosos son los estudios ${ }^{1,2}$, que han demostrado la superioridad de la angioplastía primaria como método de reperfusión en el IAM y nuestro grupo ha repor- 
tado una mortalidad a 30 días de $5,4 \%$ en pacientes con IAM y SDST sin shock cardiogénico ${ }^{7}$, resultados comparables a otras experiencias nacionales y extranjeras ${ }^{8}$. Uno de los principales determinantes del éxito de la AP es el tiempo transcurrido entre la consulta del paciente y el inflado del balón ("puerta-balón”), lo que sin duda está influenciado por la experiencia del operador y las dificultades técnicas inherentes a la anatomía vascular periférica y coronaria de los pacientes.

En esta serie no randomizada, que incluyó a todos los pacientes con IAM y elevación del ST, el éxito de la angioplastía fue de $95 \%$ y la mortalidad global a 30 días de 4,5\%. Pero nuestro mensaje es destacar que el tiempo "puerta-balón" en los pacientes explorados a través de un acceso radial fue $61 \pm 29$ minutos, similar al grupo de pacientes en los que la vía de acceso fue la arteria femoral (62 \pm 37 minutos), lo que hace de la arteria radial una excelente opción independiente de la edad de los enfermos $(62 \pm 12$ en AR vs $61 \pm 12$ años en $A F, p=n s)$. A esto se suma que el AR facilita el cuidado de los pacientes en la unidad coronaria, se acompaña de un mayor bienestar y permite una deambulación más precoz y segura, puesto que las complicaciones relacionadas al acceso son menores $(0,8 \%$ vs $4,5 \%)$.

La arteria radial es de menor tamaño y fácilmente compresible, por lo que no es difícil comprender que tenga menos complicaciones hemorrágicas, sin embargo, el rol del acceso vascular y su impacto en los resultados clínicos es escasamente difundido. Diversos estudios en los que se utilizó el acceso radial durante la angioplastía coronaria han demostrado que la reducción de las complicaciones hemorrágicas conlleva un efecto positivo en términos de mortalidad. Chase y cols ${ }^{4}$ en un estudio que incluyó sobre 30.000 pacientes sometidos a angioplastía coronaria, observaron que la vía radial se asoció a una menor necesidad de transfusión en comparación con la vía femoral (1,4 vs
$2,8 \%, \mathrm{p}<0,001)$ y a una significativa menor mortalidad a los 30 días y a 1 año (1,0\% vs $1,7 \%$ y $1,8 \%$ vs $2,9 \%$, respectivamente). Dos series publicadas recientemente que incluyó a pacientes sometidos a estudio invasivo en el contexto de un síndrome coronario agudo (SCA), confirman los resultados. En el estudio de Sciahbasi y cols, al año de seguimiento, los pacientes con acceso radial tuvieron una significativa menor incidencia de hemorragias $(0,7 \%$ vs $2,7 \%, \mathrm{p}=0,03)$ y del evento combinado de muerte o reinfarto $(4,9 \%$ vs $8,3 \%, \mathrm{p}=0,05)$ en comparación con aquellos con acceso femoral ${ }^{5}$. De igual manera otro estudio de pacientes con SCA, que comparó 509 pacientes con AR y 543 pacientes con AF, la vía radial se asoció a un $63 \%$ de reducción de riesgo de complicaciones hemorrágicas (OR 0.37; CI $0.16-0.84, \mathrm{p}=0.018$;) y a un $84 \%$ de reducción de riesgo relativo de de muerte o reinfarto intrahospitalario (OR 0.16; 95\% CI 0.03-0.62, p=0.01) ${ }^{6}$. Aún cuando en nuestra creciente experiencia las complicaciones fueron menores con el acceso radial, no hubo diferencias en términos de mortalidad al compararlos con la vía femoral, pero habrá que esperar el seguimiento alejado y el aumento de los casos.

$\mathrm{Si}$ comparamos estos resultados con las estrategias destinadas a lograr una reducción adicional en la mortalidad por infarto al miocardio, tales como la trombectomía o los nuevos antitrombóticos, podríamos decir que antes de invertir en ellas, de notable mayor costo, los centros que realicen AP debieran instaurar el acceso radial.

La trombectomía previa al implante de stent ha demostrado resultados diversos. En el estudio TAPAS no se observó una reducción de la mortalidad o el reinfarto, sólo se ha visto un efecto positivo en aquellos que logran una mejor perfusión tisular ${ }^{9}$, y un meta-análisis que incluyó 11 estudios con tromboaspiración, Burzotta y cols., demuestran una mejoría significativa de la sobrevida siendo necesario tratar 34 pacientes para 
salvar una vida ${ }^{11}$. Por otro lado, nuevos antitrombínicos tales como la bivalirudina, utilizados en el IAM han demostrado disminuir la mortalidad probablemente debido a la menor tasa de hemorragias ${ }^{3}$ pero elevando significativamente los costos del procedimiento. En conclusión, el acceso radial es tan eficaz como la vía femoral para realizar una angioplastía primaria pero con una menor tasa de complicaciones vasculares, lo que hace de ella una herramienta de fácil disponibilidad para mejorar los resultados globales del infarto agudo al miocardio sin elevar sus costos. Para su incorporación en los laboratorios de hemodinamia sólo se requiere disposición y paciencia frente a la breve curva de aprendizaje.

\section{Referencias:}

1. GUIDRY UC, EVANS JC, LARSON MG, WILSON PW, MURABITO JM, LEVY D. Temporal trends in event rates after Q-wave myocardial infarction: the Framingham Heart Study Circulation 1999;100:2054-9

2. KEELEY EC, BOURA JA, GRINES CL. Primary angioplasty versus intravenous thrombolytic therapy for acute myocardial infarction: a quantitative review of 23 randomised trials. Lancet 2003;361:13-20.

3. STONE GW, WITZENBICHLER B, GUAGLIUMI G, PERUGA JZ, BRODIE BR, DUDEK D, et al. Bivalirudin during primary PCI in acute myocardial infarction. N Engl J Med 2008; 358:2218-2230.

4. CHASE AJ, FRETZ EB, WARBURTON WP, KLINKE WP, CARERE RG, BERRY B, et al. Asociation of the arterial access site at angioplasty with transfusion and mortality: The M.O.R.T.A.L. study (Mortality benefit of reduced transfusion after percutaneous coronary intervenction via the arm or leg). Heart 2008;94:1019-1025.

5. SCIAHBASI A, PRISTIPINO C, AMBROSIO G, SPERDUTI I, SCABBIA EV, GRECO C, et al. Arterial access-site-related outcomes of patients undergoing invasive coronary procedures for acute coronary syndromes (from the ComPaRison of Early Invasive and Conservative Treatment in Patients With Non-ST-ElevatiOn Acute Coronary Syndromes [PRESTOACS] Vascular Substudy). Am J Cardiol 2009;103:796-800.

6. PRISTIPINO C, TRANI C, NAZZARO MS, BERNI A, PATTI G, PATRIZI R, et al. Major improvement of percutaneous cardiovascular procedure outcomes with radial artery catheterisation: results from the PREVAIL study. Heart 2009;95;476482.

7. PEREZ L, LECANNELIER E, OLMOS A, SALDAÑA A, VENEGAS R, PARRA J, et al. Angioplastía primaria en el infarto agudo al miocardio en el Hospital Regional de Concepción. Rev Chil Cardiol 2007;26:391-397.

8. CORBALÁN R, NAZZAL C, PRIETO J, CHÁVEZ E, LANAS F, LAMICH R, et al. Reducción de la mortalidad por infarto del miocardio en hospitales chilenos. Rev Méd Chile 2002; 130: 368-78.

9. SVILAAS T, VLAAR PJ, VAN DER HORST IC, DIERCKS GF, DE SMET BJ, VAN DER HEUVEL AF, et al. Thrombus Aspiration during Primary Percutaneous Coronary Intervention. N Engl J Med 2008; 358: 557-67.

10. BURZOTTA F, DE VITA M, GU YL, ISSHIKI T, LEFEVRE T, KALTOFT A, et al. Clinical impact of thrombectomy in acute ST-elevation myocardial infarction: an individual patient-data pooled analysis of 11 trials. Eur Heart J 2009;30:2193-203. 